\title{
Application of an electronic tongue for Tunisian olive oils' classification according to olive cultivar or physicochemical parameters
}

\author{
Souihli Slim ${ }^{1,2}$ • Nuno Rodrigues ${ }^{3,4}$ • Luís G. Dias ${ }^{1,5}$ • Ana C. A. Veloso ${ }^{6,7}$ • \\ José A. Pereira $^{8} \cdot$ Souheib Oueslati $^{2} \cdot$ António M. Peres $^{9}$
}

Received: 22 November 2016 / Revised: 10 January 2017 / Accepted: 3 February 2017 / Published online: 1 March 2017

(C) Springer-Verlag Berlin Heidelberg 2017

\begin{abstract}
Olive oil commercialization has a great impact on the economy of several countries, namely Tunisia, being prone to frauds. Therefore, it is important to establish analytical techniques to ensure labeling correctness concerning olive oil quality and olive cultivar. Traditional analytical techniques are quite expensive, time consuming and hardly applied in situ, considering the harsh environments of the olive industry. In this work, the feasibility of applying a potentiometric electronic tongue with cross-sensitivity lipid membranes to discriminate Tunisian olive oils according
\end{abstract}

António M. Peres

peres@ipb.pt

1 Escola Superior Agrária, Instituto Politécnico de Bragança, Campus Santa Apolónia, 5300-253 Bragança, Portugal

2 Institut Préparatoire aux Etudes Scientifiques et Techniques (IPEST), Laboratoire Materiaux, Molécules et Applications (LMMA), BP 51, 2070 La Marsa, Tunisia

3 REQUIMTE-LAQV/CIMO, ESA, Instituto Politécnico de Bragança, Campus Santa Apolónia, 5300-253 Bragança, Portugal

4 Departamento de Ingeniería Agrária, Universidad de Léon, Av. Portugal, No 41, 24071 Léon, Spain

5 CQ-VR, Centro de Química-Vila Real, UTAD, Apartado 1013, 5001-801 Vila Real, Portugal

6 Instituto Politécnico de Coimbra, ISEC, DEQB, Rua Pedro Nunes, Quinta da Nora, 3030-199 Coimbra, Portugal

7 CEB-Centre of Biological Engineering, University of Minho, Campus de Gualtar, 4710-057 Braga, Portugal

8 REQUIMTE-LAQV, ESA, Instituto Politécnico de Bragança, Campus Santa Apolónia, 5300-253 Bragança, Portugal

9 Laboratory of Separation and Reaction Engineering-Laboratory of Catalysis and Materials (LSRE-LCM), ESA, Instituto Politécnico de Bragança, Campus Santa Apolónia, 5300-253 Bragança, Portugal to their quality level (i.e., extra virgin, virgin or lampante olive oils) or autochthonous olive cultivar (i.e., cv Chétoui and cv Shali) was evaluated for the first time. Linear discrimination analysis coupled with the simulated annealing variable selection algorithm showed that the signal profiles of olive oils' hydroethanolic extracts allowed olive oils discrimination according to physicochemical quality level (classification model based on 25 signals enabling $84 \pm 9 \%$ correct classifications for repeated $K$-fold cross-validation), and olive cultivar (classification model based on 20 signals with an average sensitivity of $94 \pm 6 \%$ for repeated $K$-fold cross-validation), regardless of the geographical origin and olive variety or the olive quality, respectively. The results confirmed, for the first time, the potential discrimination of the electronic tongue, attributed to the observed quantitative response (sensitivities ranging from -66.6 to $+57.7 \mathrm{mV} /$ decade) of the E-tongue multi-sensors towards standard solutions of polar compounds (aldehydes, esters and alcohols) usually found in olive oils and that are related to their sensory positive attributes like green and fruity.

Keywords Electronic tongue $\cdot$ Chemometrics $\cdot$ Tunisian olive oils · Autochthonous Chétoui olive cultivar .

Autochthonous Sahli olive cultivar

\section{Introduction}

Olive oil production has a huge economic relevance in several Mediterranean countries, including Tunisia [1]. Depending on the olive cultivar, olives may be more appropriate to produce table olives or olive oil, due to different aspects like the oil content, its intrinsic physical-chemical parameters and related sensory attributes. Concerning the olive oils, the European Regulation [2] sets the values of 
physical-chemical parameters and sensory attributes used to classify olive oils according to their quality (i.e., extra virgin (EVOO), virgin (VOO) and lampante (LOO) olive oils) $[2,3]$. However, due to their commercial high value, EVOO are quite prone to frauds, namely by deliberate addition of less expensive olive oils [4]. Thus, in the last years a huge effort has been carried out to characterize olive oils namely those produced in Tunisia from autochthonous olive cultivars (e.g., Chemchali, Chemlali, Chétoui, Oueslati, Sahli, Zarrazi, Zarzis, etc.) [1, 5-8], aiming to set authentication biomarkers that could help minimize the risk of frauds. Some works reported the capability of discriminating EVOO according to the geographical origin (i.e., different regions in the same country or different countries), using different analytical techniques and chemometric tools $[4,9,10]$. In addition, Hassine et al. [11] carried out a chemical and sensorial characterization and preference mapping of autochthonous (Chetoui, Oueslati, Chemlali and Zalmati) and introduced (Arbequina) olive oil cultivars in Tunisia showing that based on those profiles it was possible to differentiate the VOO according to olive variety and geographical origin. Concerning the cultivar assessment of Tunisian olive oils, several works can be found in the literature, based on traditional analytical techniques coupled with different multivariate statistical approaches. Abdallah et al. [12] used fatty acid data obtained by direct infusion mass spectroscopy to classify Tunisian EVOO according to their genetic variety (Chemchali, Chemlali, Dhokar, Jemri, Fouji, Zalmati and Zarrazi) and maturity index. The results showed that olive oils could be correctly classified (100\%) according to olive cultivar based on linear discriminant analysis (LDA) models. These same authors [13] also demonstrated that attenuated total reflectance Fourier-transform infrared (ATR-FTIR) spectroscopy could be used to discriminate Tunisian EVOO according to olive cultivar as well as to predict olive oil mixtures, using LDA and multiple linear regression (MLR) models. The results showed that the FTIR spectra with a stepwise LDA allowed establishing a multivariate classification model that could correctly classify all EVOO according to the olive cultivar (sensitivity of $100 \%$ for both training evaluation sets). Loubiri et al. [14] used a rapid-resolution liquid chromatography coupled to electrospray ionization time-of-flight mass spectrometry (RRLC-ESI-TOF-MS) to determine the phenolic profiles of Tunisian EVOO, which were successfully used, in combination with principal component analysis, to classify olive oils according to the olive cultivar (Neb Jmal, Chétoui, Arbequina and Picholine). Essid et al. [15] verified that sensory and volatile profiles of monovarietal Tunisian EVOO could also be used to differentiate, based on PCA and clusters analysis, the geographical origin of olive oils from Chétoui cultivar, showing the influence of the geographical origin on the abovementioned parameters.
However, the majority of the analytical techniques used are time consuming, not portable and difficult to implement in situ, considering the usual harsh environments of the olive industry. Besides, due to their cost and the need of skilled technicians, these analytical tools are far away from the economic possibilities of small- and medium-scale olive oil producers. Recently researchers have focused their attention on the potential use of electrochemical devices for olive oil analysis, including quality level assessment and classification, geographical origin discrimination, olive cultivar identification as well as monitoring of olive oils physicochemical and sensorial parameters during storage [16-28]. Concerning Tunisian olive oils evaluation using electrochemical tools, to the authors' best knowledge, only one work could be found. Tena et al. [29] described the atline implementation of an electronic nose (E-nose), based on metal oxide sensors, as a tool for Tunisian VOO varietal traceability. In that work the volatile profiles of the two main Tunisian olive oils (Chemlali and Chétoui cultivars) were evaluated and, based on the responses of five E-nose sensors with PCA, it was possible to reasonably distinguish VOO samples according to the olive cultivar. In the present work, it was evaluated for the first time, the feasibility of using an electronic tongue (E-tongue) as a classification tool for discriminating Tunisian olive oils according to their quality level (i.e., EVOO, VOO and LOO, independent of the olive cultivar) and olive cultivar (i.e., Chétoui and Shali, which are two autochthonous Tunisian cultivars, regardless the quality grade). To overcome difficulties concerning the direct potentiometric analysis of olive oil, the evaluation was performed in hydroethanolic extracts supposedly rich in polar compounds, which contents are highly dependent on the olive cultivar [14, 15, 29]. Indeed, olive oils produced from a single olive cultivar (monovarietal oils) have specific physical and biochemical characteristics [12]. The potential response of the E-tongue towards polar compounds of olive oils was further checked by determining the sensitivities of each sensor towards standard solutions of chemical compounds related to sensory attributes perceived in olive oils, namely, aldehydes, esters and alcohols.

\section{Materials and methods}

\section{Olive oil samples and physicochemical analysis}

Forty-three commercial Tunisian olive oils were acquired in Tunisia, being produced in different geographical regions of the country (Table 1). During the transport, olive oil samples were kept in the original dark amber bottles, to minimize the risk of quality degradation. From those olive oils and according to the label information, 41 
Table 1 Details regarding the Tunisian olive oils: olive cultivar (according to the label information: autochthonous cv Chétoui, cv Sahli and cv El Leguim; non-autochthonous cv Arbequina and cv Koroneiki), geographical origin (according to the label information) and physicochemical quality level (EVOO, VOO and LOO, according to the physicochemical evaluation of the quality parameters following the European directives [2]; data not shown)

\begin{tabular}{|c|c|c|c|}
\hline \multirow[t]{2}{*}{ Sample number } & \multicolumn{2}{|c|}{ Label information } & \multirow{2}{*}{$\begin{array}{l}\text { Quality level (based } \\
\text { on physicochemical } \\
\text { evaluation) }^{\mathrm{c}}\end{array}$} \\
\hline & Olive cultivar $^{\mathrm{a}}$ & Geographical origin $^{\mathrm{b}}$ & \\
\hline 1 & Sahli & Sousse & LOO \\
\hline 2 & Mixed cultivars & Tunis & $\mathrm{LOO}$ \\
\hline 3 & Sahli & M'saken-Sousse & LOO \\
\hline 4 & Sahli & Sousse & LOO \\
\hline 5 & Sahli & Maremba-Monastir & LOO \\
\hline 6 & Sahli & Souse & LOO \\
\hline 7 & Sahli & Kaala-Sousse & LOO \\
\hline 8 & Chétoui & Jendouba & VOO \\
\hline 9 & Sahli & Sousse & LOO \\
\hline 10 & Sahli & Sousse & LOO \\
\hline 11 & Sahli & Sbikha-Kairouan & EVOO \\
\hline 12 & Mixed cultivars & Tunis & VOO \\
\hline 13 & Chétoui & Kef & LOO \\
\hline 14 & Sahli & Mornag-Ben Arous & EVOO \\
\hline 15 & Chétoui & Ras jebal-Bizert & LOO \\
\hline 16 & Sahli & Kasserine & EVOO \\
\hline 17 & Chétoui & Mejez el beb-Beja & LOO \\
\hline 18 & Sahli & Sousse & LOO \\
\hline 19 & Sahli & Sidi bouzid & LOO \\
\hline 20 & El Leguim & Hafouz-Kairouan & LOO \\
\hline 21 & Chétoui & Jebal resas-Nabeul & LOO \\
\hline 22 & Sahli & Kaala lekbira-Sousse & LOO \\
\hline 23 & Sahli & Mornag & LOO \\
\hline 24 & Sahli & Beja-Jendouba & LOO \\
\hline 25 & Sahli & Zaghouan & LOO \\
\hline 26 & Chétoui & Sousse & LOO \\
\hline 27 & Chétoui & Ras jebal-Bizert & LOO \\
\hline 28 & Sahli & Sebitla-Kasserine & LOO \\
\hline 29 & Chétoui & Ras jebal-Bizert & LOO \\
\hline 30 & Sahli & Teboulba-Monastir & LOO \\
\hline 31 & Sahli & Sousse & LOO \\
\hline 32 & Sahli & Sidi bouzid & LOO \\
\hline 33 & Sahli & Bouficha-Sousse & LOO \\
\hline 34 & Sahli & Kairouan & LOO \\
\hline 35 & Sahli & Takelsa-Nabeul & LOO \\
\hline 36 & Chétoui & Kelibia-Nabeul & LOO \\
\hline 37 & Chétoui & Mornag-Ben Arous & LOO \\
\hline 38 & Koroneiki & Sfax & EVOO \\
\hline 39 & Chétoui & Sfax & VOO \\
\hline 40 & Sahli & Sousse & LOO \\
\hline 41 & Sahli & Mornag & LOO \\
\hline 42 & El Leguim & Kairouan & VOO \\
\hline 43 & Arbequina & Kairouan & VOO \\
\hline
\end{tabular}

${ }^{a}$ Olive cultivars: Tunisia autochthonous cultivars (cv Chétoui, cv El Leguim and cv Sahli) and European cultivars introduced in Tunisia (cv Arbequina and cv Koroneiki)

${ }^{\mathrm{b}}$ Geographical production origin of the Tunisian olive oils under study

${ }^{c}$ Quality level according to European regulations [2] and based on the quality physicochemical contents determined in this study (data not shown): $E V O O$ extra virgin olive oil (simultaneously: FA $<0.8 \%$ oleic acid, $\mathrm{PV}<20 \mathrm{mEq} \mathrm{O}_{2} / \mathrm{kg}, K_{232}<2.50, K_{270}<0.22$ and $\Delta K<0.01$ ); $V O O$ virgin olive oil (simultaneously: FA $<2.0 \%$ oleic acid, $\mathrm{PV}<20 \mathrm{mEq} \mathrm{O}_{2} / \mathrm{kg}, K_{232}<2.60, K_{270}<0.25$ and $\Delta K<0.01$ ); or, LOO lampante olive oil (for the other cases) 
were monovarietal olive oils, the other two samples being obtained from a mixture of olives from different cultivars. Concerning the monovarietal olive oils, 39 were produced from autochthonous Tunisian olive cultivars, namely cv Sahli (26 samples), cv Chétoui (11 samples) and cv El Leguim (also called Oueslati, 2 samples); the other 2 from non-autochthonous Tunisian cultivars (European cultivars introduced in Tunisia: 1 sample from cv Arbequina and 1 sample from cv Koroneiki). Considering the number of independent olive oils gathered, only monovarietal olive oils produced from cv Chétoui and cv Sahli were used in this study to verify the feasibility of the E-tongue for olive oils classification according to autochthonous Tunisian olive cultivar. Physicochemical characterization of all olive oil samples was carried out in the laboratories of Agrarian High School of the Polytechnic Institute of Bragança (Portugal). All 43 olive oils (41 monovarietal olive oils and 2 other olive oils of unknown mixed cultivars) were analyzed following the standard methods [2] and the values of five quality parameters were quantified, namely the free acidity (FA, in \% oleic acid), the peroxide values (PV, in $\mathrm{mEq} \mathrm{O}_{2} /$ $\mathrm{kg})$ as well as the specific coefficients of extinction at 232 and $270 \mathrm{~nm}\left(K_{232}\right.$ and $K_{270}$ and $\left.\Delta K\right)$. All physicochemical assays were carried out in triplicate (i.e., three subsamples were collected from each olive oil bottle and analyzed) (data not shown). Based on the found physicochemical mean contents and according to the European Regulation [2], each of the 43 olive oils analyzed was classified (Table 1) as EVOO (if simultaneously have: FA $\leq 0.8 \%$ oleic acid, $\mathrm{PV} \leq 20 \mathrm{mEq} \mathrm{O}_{2} / \mathrm{kg}, K_{232} \leq 2.50, K_{270} \leq 0.22$ and $\Delta K \leq 0.01$ ), VOO (simultaneously: FA $\leq 2.0 \%$ oleic acid, $\mathrm{PV} \leq 20 \mathrm{mEq} \mathrm{O}_{2} / \mathrm{kg}, K_{232} \leq 2.60, K_{270} \leq 0.25$ and $\Delta K \leq 0.01$ ) or LOO (for the other cases). In addition, it should be emphasized that, for olive oils classification according to the physicochemical quality level, all the 43 olive oils were used.

\section{E-tongue device}

The E-tongue included two home-made print-screen potentiometric arrays as described by Dias et al. [16]. The electrochemical device comprised 20 sensors (diameter: $3.6 \mathrm{~mm}$; thickness: $0.3 \mathrm{~mm}$ ) corresponding to different combinations of 4 different lipid additives (octadecylamine, oleyl alcohol, methyltrioctylammonium chloride and oleic acid; $\approx 3 \%$ ); 5 different plasticizers (bis(1-butylpentyl) adipate, dibutyl sebacate, 2-nitrophenyl-octylether, tris(2ethylhexyl)phosphate and dioctyl phenylphosphonate; $\approx 65 \%$ ) and high molecular weight polyvinyl chloride (PVC; $\approx 32 \%$ ). All reagents were from Fluka (minimum purity $\geq 97 \%$ ). The type of sensors and polymeric membrane compositions (relative percentage of additive, plasticizer and PVC) were selected considering the satisfactory signal stability over time and the signal repeatability towards the basic standard taste compounds (sweet, acid, bitter, salty and umami) previously reported [30]. Lipid polymeric membranes were used since they promote interactions with taste substances via electrostatic or hydrophobic interactions [31]. Each sensor was identified with a letter $\mathrm{S}$ (for sensor) followed by a code for the sensor array (1: or 2:) and the number of the membrane (1 to 20 , corresponding to different combinations of plasticizer and additive used) as previously reported [16].

\section{E-tongue analysis}

E-tongue response towards standard solutions of gustatory olive oil molecules

The potentiometric response of the E-tongue towards standard solutions of chemical compounds, which are related to known gustatory sensations perceived in olive oils, was evaluated. Since, the direct potentiometric evaluation of olive oils is not possible due to viscosity issues, the signal profiles were recorded in water-ethanol (80:20, $\mathrm{v} / \mathrm{v})$ extracts. So, in this study, the chemical standards were also prepared in the same water-ethanol mixtures. Indeed, extracting olive oils with hydroethanolic solutions allow obtaining extracts rich in polar compounds, which are responsible for specific sensory positive attributes. So, the electrochemical response of the E-tongue versus standard solutions (concentrations ranging from $1.0 \times 10^{-5}$ to $1.1 \times 10^{-2} \mathrm{~mol} / \mathrm{L}$ in water/ethanol mixture, $80: 20 \mathrm{v} / \mathrm{v}$ ) of polar compounds usually found in olive oils have been evaluated. In each assay, the E-tongue signal profiles were recorded after a 5-min stabilization period time, aiming to give sufficient time to reach pseudo-equilibrium between the non-specific lipid polymeric membranes comprised in the electrochemical device and the chemical compound in solution. As shown in Table 2, the chemical compounds analyzed included 4-hydroxy-3-methoxybenzaldehyde or vanillin (purity of $99 \%$ from Extrasynthese), hexyl acetate (purity of 99\% from Acros Organics), (Z)-Hex-3-en-1-ol or cis-3-hexen-1-ol (98\% of purity, Sigma-Aldrich) trans hex-2-enal or $(E)$-hex-2-enal (purity of $99 \%$ from Acros Organics) and (Z)-hex-3-enyl acetate (98\% of purity, Sigma-Aldrich), which are related to different sensory attributes [29]. For instance, with the exception of vanillin, all the other chemicals have been reported in Tunisian olive oils, namely from the Chétoui variety [29]. The potentiometric response of each E-tongue sensor towards the concentration of each standard solution was evaluated by calculating the sensor sensitivity (in $\mathrm{mV} /$ decade), which is equal to the slope of the regression line between the sensor signal versus the decimal logarithm of the solution concentration. Finally, each electrochemical assay required 
Table 2 Volatile chemical compounds related to sensory attributes perceived in olive oils: name, chemical structure and main olive oil sensory attributes associated with each compound

\begin{tabular}{llll}
\hline Chemical compound (IUPAC nomenclature) & Chemical formula & Chemical family & Descriptors related to sensory attributes $^{\mathrm{a}}$ \\
\hline 4-Hydroxy-3-methoxybenzaldehyde & $\mathrm{C}_{8} \mathrm{H}_{8} \mathrm{O}_{3}$ & Phenolic aldehyde & Vanilla like sensation \\
Hexyl acetate & $\mathrm{C}_{8} \mathrm{H}_{16} \mathrm{O}_{2}$ & Ester & Sweet, green, grassy, fruity or apple sensations \\
$(Z)$-Hex-3-en-1-ol & $\mathrm{C}_{6} \mathrm{H}_{12} \mathrm{O}$ & Alcohol & Green leaves or banana sensations \\
$(E)$-Hex-2-enal & $\mathrm{C}_{6} \mathrm{H}_{10} \mathrm{O}$ & Aldehyde & Green, almonds or apple sensations \\
$(Z)$-Hex-3-enyl acetate & $\mathrm{C}_{8} \mathrm{H}_{16} \mathrm{O}_{2}$ & Ester & Fruity or green leaves sensations \\
\hline
\end{tabular}

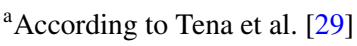

$20 \mathrm{~mL}$ of each hydroethanolic standard solution, being all solutions analyzed at controlled temperature $\left(\sim 20^{\circ} \mathrm{C}\right)$ under agitation using a magnetic stirrer (Velp Scientifica). The E-tongue was immersed directly in each solution and after 5-min stabilization period the potentiometric signal profiles the 40 E-tongue lipid membranes were acquired.

\section{Olive oil sample preparation and potentiometric assays}

Olive oils were extracted using water-ethanol solutions $(80: 20 \mathrm{v} / \mathrm{v})$, to overcome the difficulty of carrying out electrochemical assays in viscous non-conductive liquids [24] and electrochemically analyzed as previously described [16]. Ethanol was of analytical grade (Panreac, Barcelona) and deionized type II water was used in all electrochemical assays. For the electrochemical assays, samples were withdrawn from each olive oil bottle, which was previously smoothly shaken, and extracted with a solution of deionized water and ethanol (p.a.). In each assay, $10.00 \mathrm{~g}$ of olive oil was mixed to $100 \mathrm{~mL}$ of hydroethanolic solution during 5-10 min under strong agitation, using a vortex stirrer (LBX V05 series, lbx instruments), with a constant speed of approximately $500 \mathrm{rpm}$. This process allowed the extraction of polar compounds, which are related to sensory sensations of olive oils [17]. The mixture was left at ambient temperature during $60 \mathrm{~min}$, after which, $40.0 \mathrm{~mL}$ of the supernatant solution was carefully removed and immediately analyzed with the E-tongue, during 5 min enabling to carry out several electrochemical scans, with the last one retained, which would correspond to a pseudo-equilibrium state. The analyses were performed in duplicate, using the electrochemical "average" signal profile per olive oil in the data treatment (assumed as the olive oil-specific fingerprint) to establish a more representative sample profile of potentiometric signals.

\section{Statistical analysis}

Linear discriminant analysis (LDA) was applied to infer about the capability of the E-tongue device to discriminate standard hydroethanolic solutions of polar compounds usually found in olive oils and related their positive sensory attributes, allowing inference about the potentiometric nonspecific response of the E-tongue lipid membranes towards these chemical compounds. In addition, LDA was applied to verify the potential of the electrochemical multi-sensor device to classify edible and inedible monovarietal Tunisian olive oils according to autochthonous olive cultivars, regardless of their geographical origin, or to distinguish olive oils according to their physicochemical quality level, independent of the olive cultivar used in their production or the geographical origin. In these studies, it was necessary to select the most informative sensors (subset of independent predictors) to be included in the final LDA models, since not all sensors present relevant information and so their inclusion in the classification models may increase the noise effects. The best subsets of sensors were established between 40 potentiometric sensor signals using a metaheuristic simulated annealing (SA) variable selection algorithm [32-34]. The LDA potential was evaluated using two cross-validation (CV) variants: leave-one-out (LOO-CV), known to be an overoptimistic procedure, and repeated $K$-fold (repeated $K$-fold-CV) technique. For the latter, data were randomly split into $K$-folds, with each of the folds left out in turn and the other $K-1$ folds used to train the model. The held out fold was used for test purposes and the quality of the predictions was assessed using the percentage of correct classifications. The $K$-estimates are averaged to get the overall resampled estimate [35]. In this work, the $K$-folds were set equal to 4 , enabling the random formation of internal validation subsets (for each gustatory group) with $25 \%$ of the initial data, allowing bias reduction. The procedure was repeated 10 times for putting the model under stress. The repeated $K$-fold- $\mathrm{CV}$ technique allows reducing the uncertainty of the estimates, by evaluating the predictive performance of the models established using $4 \times 10$ random subsets for internal validation (i.e., 40 total resamples). To normalize the weight of each variable in the final linear classification model, variable scaling and centering procedures were evaluated. The classification performance of each LDA model was graphically evaluated using a 2-D plot of the two first discriminant functions (when more than 
two class groups were considered) or by plotting the 1-D frequency distribution of the data for the sole discriminant function, for the cases where only two classes were evaluated. All statistical analysis were performed using the Subselect [33, 36] and MASS [37] packages of the open-source statistical program $\mathrm{R}$ (version 2.15.1), at a 5\% significance level.

\section{Results and discussion}

\section{E-tongue sensitivity towards polar compounds related to olive oils' sensory positive attributes}

The E-tongue performance towards polar compounds usually found in olive oils and that are related to their sensory attributes (e.g., green, sweet, fruity) was evaluated based on the sensors' quantitative response in the presence of standard aqueous-ethanol solutions with different concentrations of chemical compounds comprising aldehydes, alcohols and esters (Table 2). Indeed, it has been reported that olive oils' polar compounds, which can be extracted using hydroethanolic solutions, are mainly responsible for bitterness, astringency and pungency sensations [24, 38-40]. The plots of the potentiometric signal profiles recorded with each E-tongue sensor versus the decimal logarithm of the concentrations of each standard solution showed that in general all the different lipid polymeric membranes (combinations of 4 additive compounds and 5 plasticizers) that comprised the E-tongue arrays (20 sensors and respective replicas) showed a linear response $\left(0.9002 \leq R^{2} \leq 0.9993\right)$ towards one or more of the standard compounds analyzed, demonstrating the hypothesis previously discussed by the research team [16, 18]. Indeed, Dias et al. [16, 18] had attributed to the E-tongue capacity for correctly classifying monovarietal Portuguese and Spanish EVOOs according to the olive cultivar to the ability of the lipid membranes of the multi-sensor device, to show different potentiometric responses towards specific polar compounds and their concentrations, which are usually found in olive oils and may be extractable with hydroethanolic solutions. Briefly,

1. for 4-hydroxy-3-methoxybenzaldehyde (vanillin) the sensitivity values ranged from -12.7 to $-4.1 \mathrm{mV} /$ decade and from +2.4 to $+38.0 \mathrm{mV} /$ decade $\left(0.9022 \leq R^{2} \leq 0.9993\right)$, with the exception of the following sensors (S1:5, S1:9 and S1:10) and respective sensor replicas (S2:5, S2:9 and $\mathrm{S} 2: 10)$;

2. for $(E)$-hex-2-enal the sensitivities calculated varied from -16.8 to $-6.9 \mathrm{mV} /$ decade and from +4.2 to $+69.3 \mathrm{mV} /$ decade $\left(0.9022 \leq R^{2} \leq 0.9993\right)$, for all the sensors with the exception of S1:16 and its replica (S2:16);
3. for (Z)-hex-3-en-1-ol sensitivities ranged from -66.6 to $-5.61 \mathrm{mV} / \mathrm{dec}$ ade and from +3.50 to $+57.7 \mathrm{mV} /$ decade $\left(0.9002 \leq R^{2} \leq 0.9950\right)$, with the exception of sensors $\mathrm{S} 1: 4$ to $\mathrm{S} 1: 6, \mathrm{~S} 1: 10$ and $\mathrm{S} 1: 20$, and respective replicas (S2:4 to S2:6, S2:10 and S2:20);

4. for hexyl acetate, sensitivities varied from -6.1 to $-4.2 \mathrm{mV} /$ decade and +1.5 to $+15.3 \mathrm{mV} /$ decade $\left(0.9020 \leq R^{2} \leq 0.9957\right)$ with the exception of the sensors $\mathrm{S} 1: 3, \mathrm{~S} 1: 4, \mathrm{~S} 1: 10-\mathrm{S} 1: 12$ and $\mathrm{S} 1: 20$, and respective replicas (S2:3, S2:4, S2:10 to $\mathrm{S} 2: 12$ and $\mathrm{S} 2: 20$ );

5. for (Z)-hex-3-enyl acetate sensitivities ranged from $+2.1 \mathrm{mV} /$ decade to $+52.8 \mathrm{mV} /$ decade $\left(0.9001 \leq R^{2} \leq 0.9859\right)$, for all the sensors except $\mathrm{S} 1: 4$ and its replica (S2:4).

The study allowed to verify that for esters, the sensors and replicas mainly showed positive sensitivity values. For aldehydes and alcohol compounds, sensors and replicas displayed both positive and negative sensitivities, with the last polar compound showing the highest range of sensitivities. In addition, it was observed that some sensors and respective replicas of the E-tongue (lipid membrane applied, using the drop-by-drop technique, in the first array or in the second array of the E-tongue) had different electrochemical behaviors towards the same polar compound studied. In some cases, both sensor and replica had the same behavior (positive or negative signal decrease with the increasing concentration of the target molecule) with similar sensitivities or with quite different magnitudes. For other sensor-replica pairs, only the sensor or the replica showed a potentiometric response towards the same analyte. Finally, in fewer situations, sensor and replica had different signal trend behaviors (positive and negative) resulting in a positive or negative sensitivity values for a specific polar compound. Based on these findings, it can be concluded that, in general, both sensor and sensor replica should be considered as independent sensors due to the probably slight different physical properties (e.g., transparency, porosity, permeability) that may occur due to the possible formation of inhomogeneous membrane surfaces, which could be attributed to the drop-by-drop membrane preparation technique [41]. The differences in the membrane surfaces of sensor and sensor replica may result in the occurrence of different adsorption phenomena and surface chemical reactions, which may lead to deviations in sensors' readings [42]. Indeed, the differences of the signal profiles observed for some sensor replica comprised in the E-tongue, tentatively attributed to the manually home-made procedure used in the sensor-array construction, could be minimized in the future since monitored and controlled production procedure is envisaged. In addition, it should be remembered that the practical application of this kind of electrochemical sensor arrays would require a daily calibration routine 
step [42], a common practice when potentiometric-based analytical techniques are applied (e.g., similar to the use of $\mathrm{pH}$ electrodes), allowing minimizing the risk of drift signals effects.

Moreover, as expected, from the sensitivity results it can also be stated that, with the exception of few some punctual cases, the overall E-tongue sensors are poorly selective (for most sensors, low sensitivities ranging from -10 to $+10 \mathrm{mV} /$ decade), partially specific (cross-sensitivity) and non-specific, with the majority of them showing a potentiometric response towards all the polar compounds evaluated, but not to any in particular.

At last, the potentiometric signal profiles recorded for the different chemical compounds evaluated (i.e., alcohol, esters and aldehydes), related to sensory positive attributes of olive oils (Table 2), were used to verify if the E-tongue could discriminate different polar compounds that can be found in olive oil samples. The study carried out showed that an E-tongue-LDA-SA model, with two discriminant functions (explaining 98.1 and $1.7 \%$ of the original data variability, respectively) and based on five sensor signals (first E-tongue array: S1:12, S1:15 and S1:18; second E-tongue array: S2:17 and S2:18), could correctly separate the five different chemical standard solutions (concentrations from $1 \times 10^{-5}$ to $1.13 \times 10^{-2} \mathrm{~mol} / \mathrm{L}$ ) studied with sensitivities of $100 \%$, for both original grouped data (Fig. 1) and LOO-CV procedure. Furthermore, the discrimination capability may be tentatively attributed to the differences of sensory attributes related to each compound (Fig. 1). The discrimination predictive performance achieved with the E-tongue-LDA-SA model was further tested by implementing a repeated $K$-fold-CV procedure (four folds $\times 10$ repetitions), which minimizes the risk of model overfitting as well as the possibility of obtaining overoptimistic results, as previously discussed. The E-tongue-LDA-SA model based on the same five sensors allowed obtaining an average sensitivity of $100 \%$, for all the data randomly split. This fact strengthens the hypothesis that the E-tongue had a different potentiometric behavior depending on the polar compound, which could be accessed through chemometric techniques.

\section{Electrochemical evaluation of Tunisian olive oils using an E-tongue}

\section{Tunisian olive oils potentiometric signal profiles}

Different potentiometric signal profiles (varying from $-71 \mathrm{mV}$ to $+291 \mathrm{mV}$ ) were acquired by the E-tongue sensors (20 different lipid polymeric membranes in duplicate: S1:1-S1:20 and S2:1-S2:20) during the electrochemical analysis of the olive oils' hydroethanolic extracts depending on the physicochemical quality of the olive oils (i.e., EVOO, VOO or LOO) and on the olive

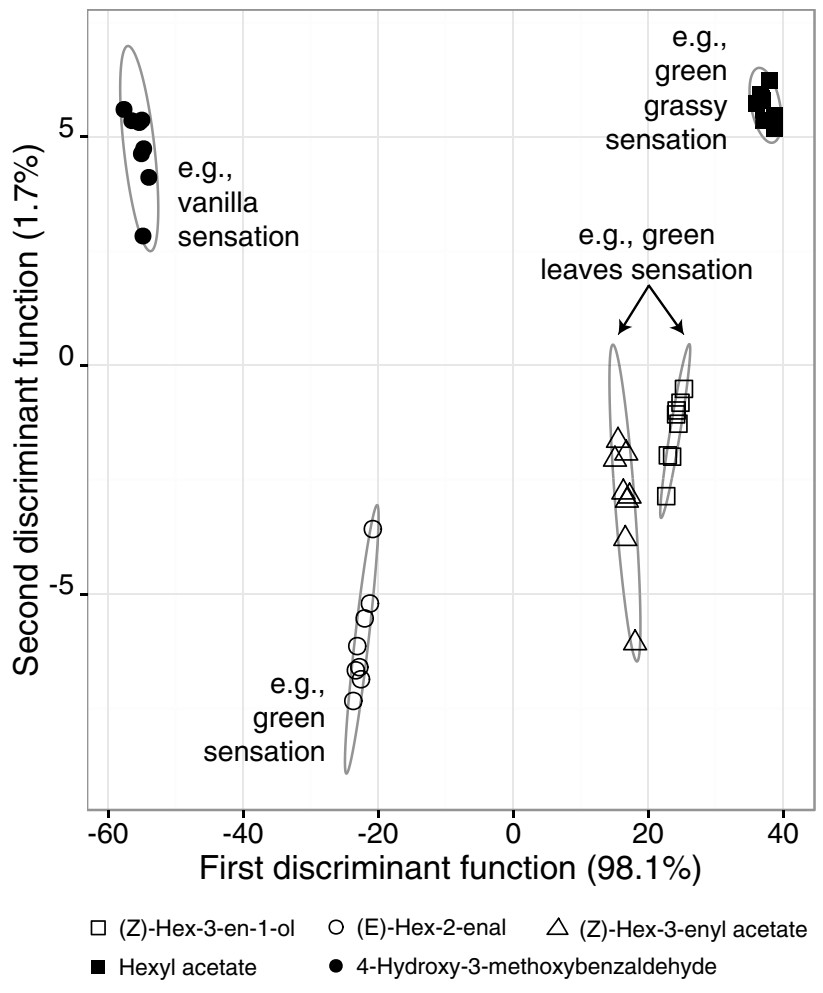

Fig. 1 Discrimination of hydroethanolic standard solutions of polar compounds (alcohols, aldehydes and esters) present in olive oils, which are related to sensory positive attributes (e.g., vanilla, green, green leaves and green grassy), using an E-tongue-LDA-SA model based on the potentiometric information gathered by five sensors (S1:12, S1:15, S1:18, S2:17 and S2:18): 4-hydroxy-3-methoxybenzaldehyde (or vanillin), hexyl acetate, $(Z)$-hex-3-en-1-ol (or cis-3-hexen1-ol), (E)-hex-2-enal and (Z)-hex-3-enyl acetate

cultivar (i.e., cv Chétoui and cv Sahli). Figure 2 shows the potentiometric average signals ( \pm standard deviation) for each sensor and for the monovarietal Tunisian olive oils from cv Chétoui and cv Sahli, regardless of their physicochemical quality grade or the geographical origin. From the observation of the plot it can be concluded that olive oils produced from the two Tunisian autochthonous cultivars have similar average E-tongue signal trend profiles. However, slight differences can be observed, namely in the signals' intensities as well as in the potentiometric signal dynamic ranges, which may allow the successful discrimination of these two monovarietal olive oils based on the electrochemical fingerprints of the hydroethanolic extracts. A similar situation was found for olive oils with different physicochemical quality grades, as can be observed from Fig. 3, where the average signal profiles ( \pm standard deviation) according to each physicochemical quality level (EVOO, VOO or LOO) are plotted, independent of the olive cultivar or geographical origin. 

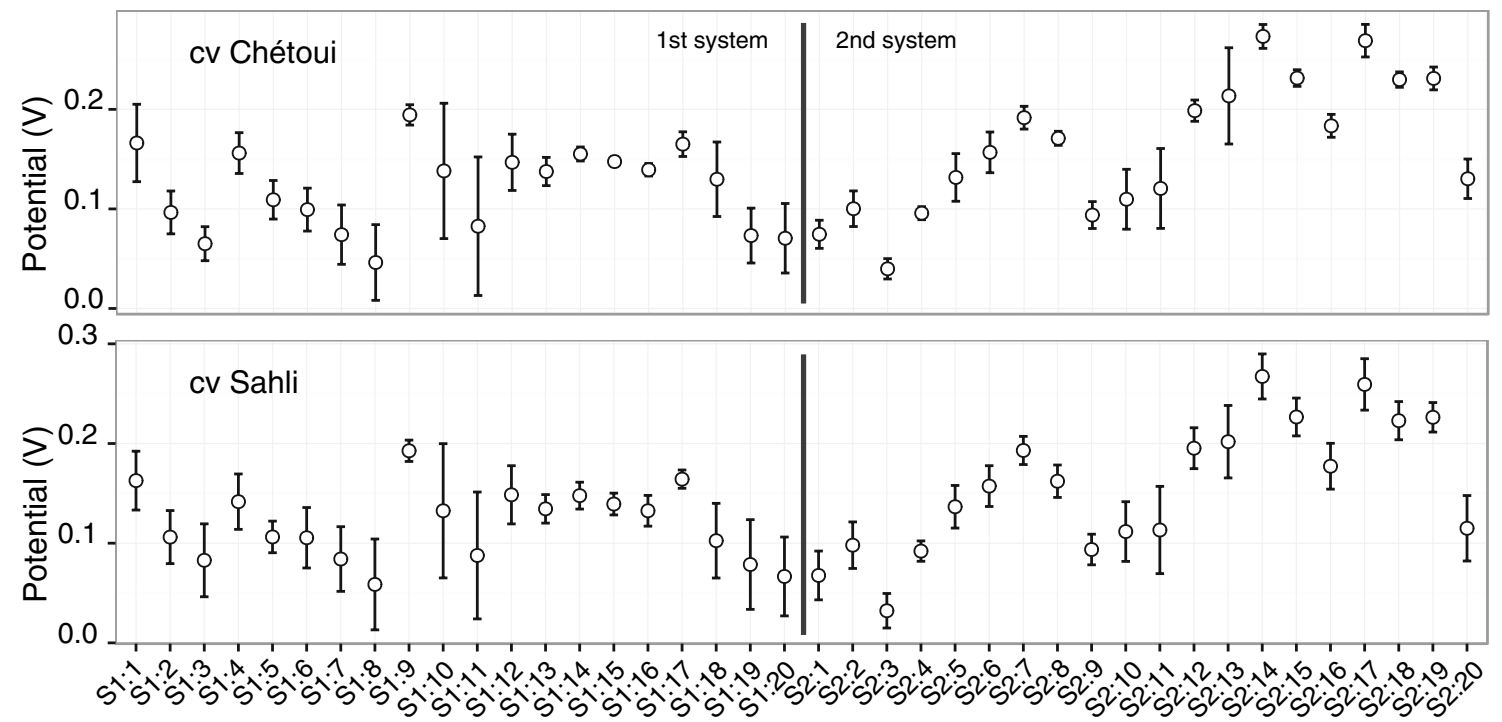

Sensor identification

Fig. 2 Potentiometric signal profile plots (average signal \pm standard deviation) for each sensor and monovarietal Tunisian olive oils according to the autochthonous olive cultivar: cv Chétoui and cv Sahli
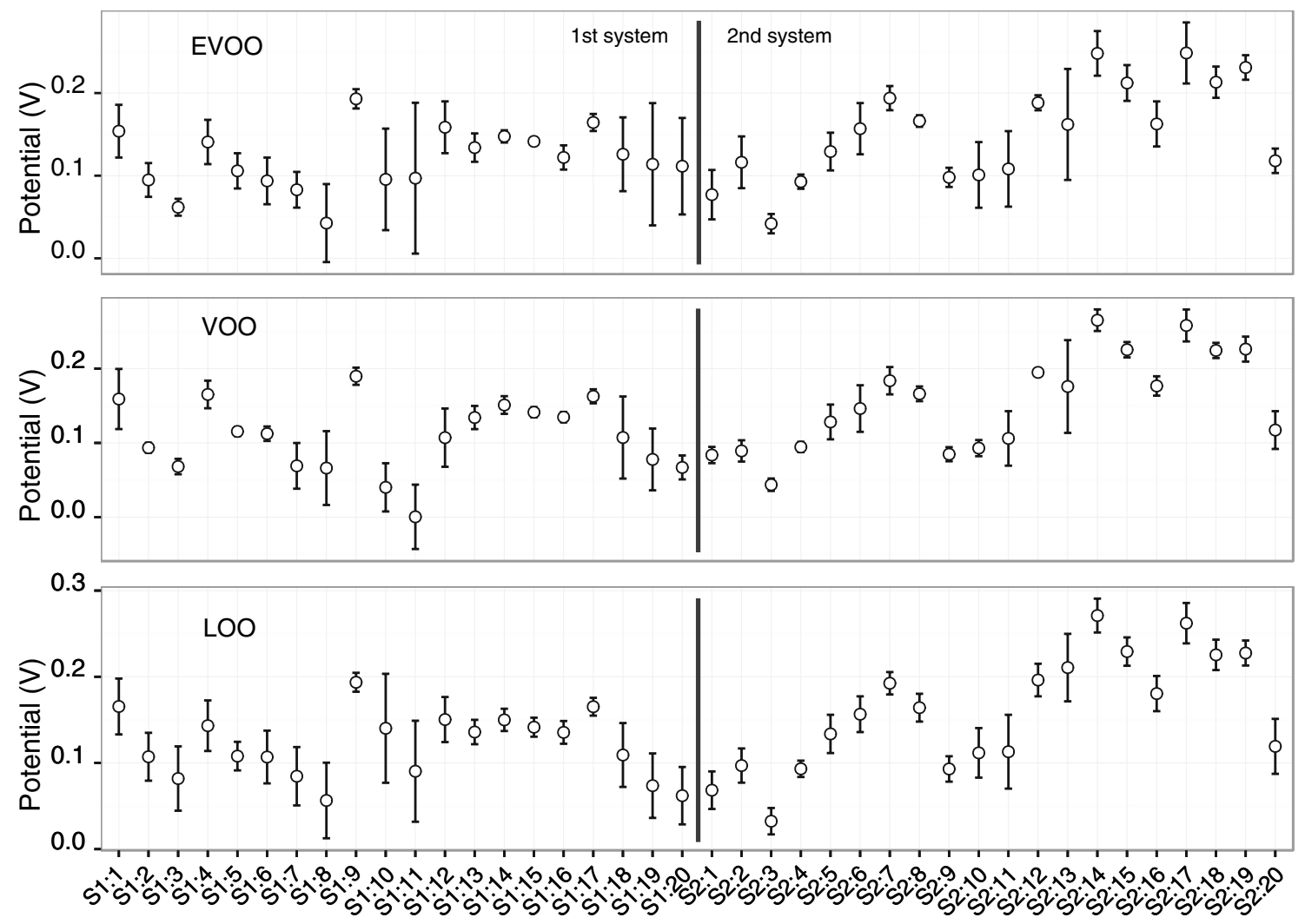

Sensor identification

Fig. 3 Potentiometric signal profile plots (average signal \pm standard deviation) for each sensor and Tunisian olive oils according to the physicochemical quality grade: EVOO (extra virgin olive oils), VOO (virgin olive oils) and LOO (lampante olive oils) 
Tunisian olive oils' physicochemical quality discrimination based on electrochemical profiles

According to the physicochemical analysis (FA, PV, $K_{232}$, $K_{270}$ and $\Delta K$ values, data not shown) and following the European regulations [2], the 43 olive oils evaluated in this work and produced in different Tunisian geographical regions from autochthonous olive cultivars (cv Chétoui, cv Sahli and cv El Leguim) and introduced European olive varieties (cv Arbequina and cv Koroneiki) were classified as EVOO (4 samples), VOO (5 samples) and LOO (34 samples) (Table 1).

For classification purposes, it was possible to establish an E-tongue-LDA-SA model with two discriminant functions that explained $100 \%$ of the original data variability (94.8 and $5.2 \%$, respectively) based on potentiometric profiles of 25 E-tongue sensors ( $\mathrm{S} 1: 2$ to $\mathrm{S} 1: 5, \mathrm{~S} 1: 8$, $\mathrm{S} 1: 10$ to $\mathrm{S} 1: 13, \mathrm{~S} 1: 15$ to $\mathrm{S} 1: 17, \mathrm{~S} 1: 20, \mathrm{~S} 2: 1$ to $\mathrm{S} 2: 6$, S2:9, S2:14 to S2:18), selected by the SA algorithm. The SA variable selection algorithm enabled the inclusion of repeated sensors in the discrimination model (7 sensors with the same membrane composition and the remaining 18 with different membrane composition), in agreement with the conclusion of Correia et al. [43], which reported the advantage of including repeated sensor signals for improving the global performance of multivariate models. On the other hand, as discussed in the previous section, the sensor-replica pairs may be treated as independent sensors regardless of the similar composition of the lipid polymeric membranes. Moreover, it should be remarked that four (i.e., S1:12, S1:15, S2:17 and S2:18) of the five E-tongue sensors that showed greater qualitative discrimination potential of polar compounds usually found in olive oils were also included in the E-tongueLDA-SA model established for classifying the olive oil samples according to their physicochemical quality. This fact supports the hypothesis previously proposed by the research team $[16,18]$ that the discrimination potential of the E-tongue may be attributed to the capability of generating different potentiometric signal profiles depending on the type and levels of olive oils' polar compounds extracted with the hydroethanolic solution. The proposed model allowed $100 \%$ correct classification of the original data, i.e., of the Tunisian olive oils according to their quality level, regardless the olive cultivar used in their production and the geographical origin (Fig. 4) and $88.4 \%$ predictive correct classification for the LOO$\mathrm{CV}$. The LOO-CV results showed that no EVOO or VOO were misclassified as LOO, being the main misclassification issue between the two first categories. This fact, together with the results plotted in Fig. 4, clearly indicates that the first discriminant function of the E-tongueLDA-SA model allows distinguishing edible (EVOO and

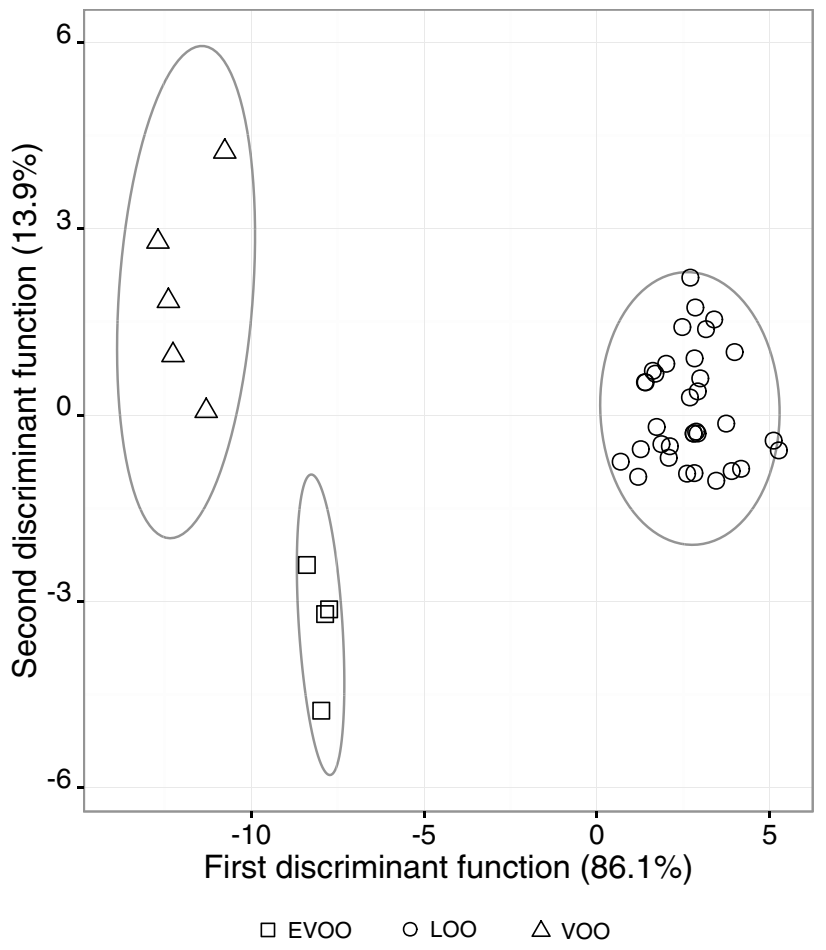

Fig. 4 Tunisian olive oils' quality level discrimination using an E-tongue-LDA-SA model (first and second discriminant functions) based on 25 potentiometric signals recorded during the analysis of hydroethanolic extracts (first array: $\mathrm{S} 1: 2$ to $\mathrm{S} 1: 5, \mathrm{~S} 1: 8, \mathrm{~S} 1: 10$ to $\mathrm{S} 1: 13, \mathrm{~S} 1: 15$ to S1:17, S1:20; second array: S2:1 to S2:6, S2:9, S2:14 to $\mathrm{S} 2: 18$ )

VOO) from inedible (LOO) Tunisian olive oils, regardless of the olive cultivar and geographical production region.

The predictive performance of the E-tongue-LDA-SA model to correctly classify the Tunisian olive oils according to their physicochemical quality was further evaluated using a repeated $K$-fold-CV procedure (four folds $\times 10$ repetitions), which allowed to retain $25 \%$ of the data for internal validation in each of the 40 random data split. The best predictive classification results were also achieved with a model established on the same 25 E-tongue sensors. In this case, an average correct classification of $84 \pm 9 \%$ (varying from 70 to $100 \%$ for the 40 evaluations performed during the repeated $K$-fold-CV procedure). Although, the predictive sensitivity is not as high as desirable, it is quite satisfactory taking into account that the studied olive oils included monovarietal (41 samples) and multivarietal (2 samples) olive oils, produced in different geographical regions of Tunisia from different olive cultivars, including autochthonous olive cultivars (cv Chéthoui, cv Sahli and cv El Leguim) and the olive cultivars introduced from Europe (cv Arbequina and cv Koroneiki). So, the potential of the potentiometric E-tongue to classify Tunisian olive oils as 
EVOO, VOO and LOO was verified based on the electrochemical profiles recorded in olive oils' hydroethanolic extracts, and specially the capability to correctly discriminate between edible and inedible olive oils. To the authors' best knowledge, this potential was only previously demonstrated for voltammetric E-tongues [25, 26, 44].

\section{Monovarietal Tunisian olive oil discrimination based on electrochemical profiles}

As it can be observed from Table 1, among the 43 Tunisian olive oils, only 41 samples were monovarietal olive oils and for these, it was only possible to have more than two olive oils for two olive cultivars: cv Chétoui (11 samples) and cv Sahli (26 samples). Then, the capability of the potentiometric E-tongue to discriminate monovarietal olive oils regardless of their quality level (EVOO, VOO or LOO) was only evaluated considering those two autochthonous olive cultivars, which are classified as Ancient Olive Orchards (AOOs), with the first mainly cultivated in the north of Tunisia and the latter in the Tunisian coast and south governorates.

An E-tongue-LDA-SA model with one discriminant function was established based on potentiometric profiles of 20 E-tongue sensors (S1:3 to S1:6, S1:8 to S1:11, S1:13 to $\mathrm{S} 1: 15, \mathrm{~S} 1: 17$ to $\mathrm{S} 1: 20, \mathrm{~S} 2: 5, \mathrm{~S} 2: 6, \mathrm{~S} 2: 10, \mathrm{~S} 2: 15$ and S2:20), also selected using the SA algorithm, turning out in the inclusion of repeated sensors (5 sensors with the same membrane composition and the remaining 15 with different membrane composition). Once again, four (i.e., S1:15, S1:18, S2:17 and S2:18) of the five E-tongue sensors that showed greater qualitative discrimination potential towards the polar compounds evaluated in this study were also included in the E-tongue-LDA-SA model established for classifying the olive oil samples according to their olive cultivar. The proposed model allowed $97.3 \%$ correct classifications of the original data (Fig. 5), i.e., of the Tunisian olive oils according to the olive cultivar, which included both edible (EVOO and VOO) and inedible (LOO) olive oils produced in different Tunisian geographical regions. Moreover, for the LOO-CV technique, the olive cultivar (cv Chétoui or cv Sahli) of $94.6 \%$ of the olive oils could be correctly predicted.

The predictive capability of the E-tongue classification model was further assessed by applying the $K$-fold-CV procedure (four folds $\times 10$ repetitions). For this more realistic CV variant the E-tongue-LDA-SA model based on the same 20 sensor signals allowed obtaining a mean sensitivity of $94 \pm 6 \%$ (varying from 78 to $100 \%$ for the 40 runs carried out during the repeated $K$-fold-CV procedure). The results confirmed the potential of the potentiometric E-tongue to classify monovarietal Tunisian olive oils according to their autochthonous olive cultivar, regardless

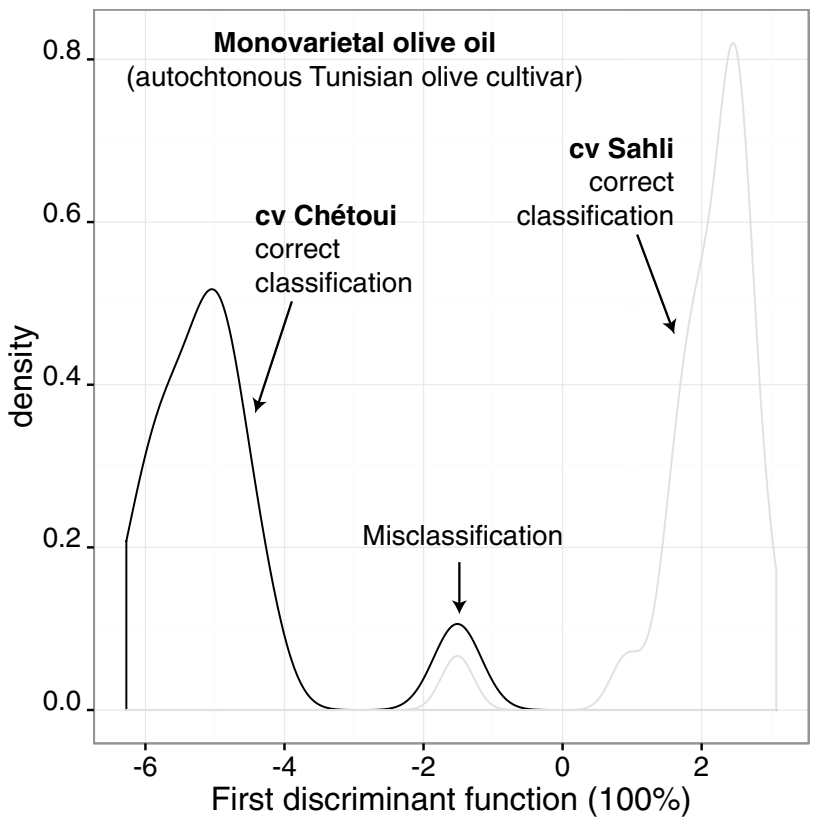

Fig. 5 Frequency distribution (one-dimension plot) of the data for the discriminant function of the E-tongue-LDA-SA classification model based on 20 potentiometric signals (first array: S1:3 to S1:6, $\mathrm{S} 1: 8$ to $\mathrm{S} 1: 11, \mathrm{~S} 1: 13$ to $\mathrm{S} 1: 15$ and $\mathrm{S} 1: 17$ to $\mathrm{S} 1: 20$; second array: $\mathrm{S} 2: 5$, $\mathrm{S} 2: 6, \mathrm{~S} 2: 10, \mathrm{~S} 2: 15$ and $\mathrm{S} 2: 20)$ recorded during the analysis of hydroethanolic extracts of Tunisian monovarietal olive oils produced from two autochthonous cultivars (cv Chétoui and cv Sahli)

of the physicochemical quality level. Previously, this E-tongue capability was already described by the research team for monovarietal Portuguese and Spanish olive oils $[16,18]$, although in those cases only EVOO were considered, which were produced in only two limited geographical regions (one in Portugal and the other in Spain).

\section{Conclusions}

In this work, it was shown that a potentiometric E-tongue comprising 20 lipid polymeric membranes (and respective replicas) as sensor units possessed non-specific electrochemical qualitative and quantitative electrochemical responses towards polar compounds usually found in the chemical composition of olive oils and that are related to some of their positive sensory attributes, such as green and fruity sensations. Furthermore, it was demonstrated that the E-tongue signal profiles recorded during the analysis of olive oils' hydroethanolic extracts, which may contain high levels of different polar compounds, could be foreseen as a practical overall electrochemical fingerprint of Tunisian olive oils. Indeed, the versatile potentiometric information gathered by the E-tongue enabled a successful classification of Tunisian monovarietal olive oils according to two autochthonous olive cultivars (cv Chétoui and cv Sahli) 
used in the production, regardless of their physicochemical quality grade and geographical origin, as well as the satisfactory discrimination of edible from inedible olive oils (i.e., extra virgin, virgin or lampante olive oils), regardless of the olive cultivar used or the geographical origin. For the first time, the practical usefulness of this type of electrochemical multi-sensor device for Tunisian olive oils evaluation is described, showing its potential for minimizing the risk of possible frauds, namely those related with olive oil mislabeling and so, to strengthen the Tunisian olive oil recognition.

Acknowledgements This work was financially supported by Project POCI-01-0145-FEDER-006984-Associate Laboratory LSRE-LCM and by Project UID/QUI/00616/2013-CQ-VR both funded by FEDER - Fundo Europeu de Desenvolvimento Regional through COMPETE2020-Programa Operacional Competitividade e Internacionalização (POCI) — and by national funds through FCTFundação para a Ciência e a Tecnologia, Portugal. Strategic funding of UID/BIO/04469/2013 unit is also acknowledged. Nuno Rodrigues thanks FCT, POPH-QREN and FSE for the Ph.D. Grant (SFRH/ BD/104038/2014).

\section{Compliance with ethical standards}

Conflict of interest Souihli Slim declares that he has no conflict of interest. Nuno Rodrigues declares that he has no conflict of interest. Luís G. Dias declares that he has no conflict of interest. Ana C.A. Veloso declares that she has no conflict of interest. José A. Pereira declares that he has no conflict of interest. Souheib Oueslati declares that he has no conflict of interest. António M. Peres declares that he has no conflict of interest.

Ethical approval This article does not contain any studies with human participants or animals performed by any of the authors.

Informed consent Not applicable.

\section{References}

1. Dabbou S, Issaoui M, Esposto S, Sifi S, Taticchi A, Servili M, Montedoro GF, Hammami M (2009) Cultivar and growing area effects on minor compounds of olive oil from autochthonous and European introduced cultivars in Tunisia. J Sci Food Agric 89:1314-1325

2. Anonymous (1991) Commission regulation (ECC) no 2568/91 and all subsequent amendments: on the characteristics of olive oil and olive-pomace oil and on the relevant methods of analysis. Off J Eur Union L248:1-82

3. Ortega JB, Gila DMM, Puerto DA, García JG, Ortega JG (2016) Novel technologies for monitoring the in-line quality of virgin olive oil during manufacturing and storage. J Sci Food Agric 96:4644-4662

4. Bazakos C, Khanfir E, Aoun M, Spano T, El Zein Z, Chalak L, El Riachy M, Abou-Sleymane G, Ali SB, Kammoun NG, Kalaitzis P (2016) The potential of SNP-based PCR-RFLP capillary electrophoresis analysis to authenticate and detect admixtures of Mediterranean olive oils. Electrophoresis 37:1881-1890
5. Dabbou S, Brahmi F, Taamali A, Issaoui M, Ouni Y, Braham M, Zarrouk M, Hammami M (2010) Extra virgin olive oil components and oxidative stability from olives grown in Tunisia. J Am Oil Chem Soc 87:1199-1209

6. Ouni Y, Flamini G, Zarrouk M (2016) The Chemical properties and volatile compounds of virgin olive oil from Oueslati variety: influence of maturity stages in olives. J Am Oil Chem Soc 93:1265-1273

7. Fares N, Jabri IK, Sifi S, Abderrabba M (2016) Physical chemical and sensory characterization of olive oil of the region of Kairouan. J Mater Environ Sci 7:2148-2154

8. Issaoui M, Dabbou S, Echbili A, Rjiba I, Gazzah N, Trigui A, Hammami M (2007) Biochemical characterisation of some Tunisia virgin olive oils obtained from different cultivars growing in Sfax National Collection. J Food Agric Environ $5: 17-21$

9. Giacalone R, Giuliano S, Gulotta E, Monfreda M, Presti G (2015) Origin assessment of EV olive oils by esterified sterols analysis. Food Chem 188:279-285

10. Laroussi-Mezghani S, Vanloot P, Molinet J, Dupuy N, Hammami M, Grati-Kamoun N, Artaud J (2015) Authentication of Tunisian virgin olive oils by chemometric analysis of fatty acid compositions and NIR spectra. Comparison with Maghrebian and French virgin olive oils. Food Chem 173:122-132

11. Hassine KB, Taamalli A, Slama MB, Khouloud T, Kiristakis A, Benincasa C, Perri E, Malouche D, Hammami M, Bornaz S, Grati-Kammoun N (2015) Characterization and preference mapping of autochthonous and introduced olive oil cultivars in Tunisia. Eur J Lipid Sci Technol 117:112-121

12. Abdallah M, Vergara-Barberán M, Lerma-García MJ, HerreroMartínez JM, Simó-Alfonso EF, Guerfel M (2016) Classification of Tunisian extra virgin olive oils according to their genetic variety and maturity index using fatty acid profiles established by direct infusion mass spectrometry. Eur J Lipid Sci Technol 118:735-743

13. Abdallah M, Vergara-Barberán M, Lerma-García MJ, HerreroMartínez JM, Simó-Alfonso EF, Guerfel M (2016) Cultivar discrimination and prediction of mixtures of Tunisian extra virgin olive oils by FTIR. Eur J Lipid Sci Technol 118:1236-1242

14. Loubiri A, Taamalli A, Talhaoui N, Mohamed SN, Carretero AS, Zarrouk M (2017) Usefulness of phenolic profile in the classification of extra virgin olive oils from autochthonous and introduced cultivars in Tunisia. Eur Food Res Technol 243:467-479. doi:10.1007/s00217-016-2760-7

15. Essid F, Sifi S, Beltrán G, Sánchez S, Raïes A (2016) Sensory and volatile profiles of monovarietal North Tunisian extra virgin olive oils from 'Chétoui' cultivar. J Oleo Sci 65:533-542

16. Dias LG, Fernandes A, Veloso ACA, Machado AASC, Pereira JA, Peres AM (2014) Single-cultivar extra virgin olive oil classification using a potentiometric electronic tongue. Food Chem 160:321-329

17. Veloso ACA, Dias LG, Rodrigues N, Pereira JA, Peres AM (2016) Sensory intensity assessment of olive oils using an electronic tongue. Talanta 146:585-593

18. Dias LG, Rodrigues N, Veloso ACA, Pereira JA, Peres AM (2016) Monovarietal extra virgin olive oils classification: a fusion of human sensory attributes and an electronic tongue. Eur Food Res Technol 242:259-270

19. Rodrigues N, Dias LG, Veloso ACA, Pereira JA, Peres AM (2016) Monitoring olive oils quality and oxidative resistance during storage using an electronic tongue. LWT Food Sci Technol 73:683-692

20. Rodrigues N, Dias LG, Veloso ACA, Pereira JA, Peres AM (2016) Evaluation of extra-virgin olive oils shelf life using an electronic tongue-chemometric approach. Eur Food Res Technol. doi:10.1007/s00217-016-2773-2 
21. Rodríguez-Méndez ML, Apetrei C, de Saja JA (2010) In: Preedy VR, Watson RR (eds) Olives and olive oil in health and disease prevention. Academic Press, London

22. Apetrei C, Ghasemi-Vernamkhasti M, Apetrei IM (2016) In: Rodríguez-Méndez ML (ed) Electronic noses and tongues in food science. Academic Press, London

23. Apetrei IM, Apetrei C (2013) Voltammetric e-tongue for the quantification of total polyphenol content in olive oils. Food Res Int 54:2075-2082

24. Apetrei C, Apetrei IM, Villanueva S, de Saja JA, GutierrezRosales F, Rodriguez-Mendez ML (2010) Combination of an e-nose, an e-tongue and an e-eye for the characterisation of olive oils with different degree of bitterness. Anal Chim Acta 663:91-97

25. Oliveri P, Baldo MA, Daniele S, Forina M (2009) Development of a voltammetric electronic tongue for discrimination of edible oils. Anal Bioanal Chem 395:1135-1143

26. Cosio MS, Ballabio D, Benedetti S, Gigliotti C (2006) Geographical origin and authentication of extra virgin olive oils by an electronic nose in combination with artificial neural networks. Anal Chim Acta 567:202-210

27. Haddi Z, Alami H, El Bari N, Tounsi M, Barhoumi H, Maaref A, Jaffrezic-Renault N, Bouchikhi B (2013) Electronic nose and tongue combination for improved classification of Moroccan virgin olive oil profiles. Food Res Int 54:1488-1498

28. Cimato A, Dello-Monaco D, Distante C, Epifani M, Siciliano P, Taurino AM, Zuppa M, Sani G (2006) Analysis of singlecultivar extra virgin olive oils by means of Electronic Nose and HSSPME/GC/MS methods. Sens Actuator B 114:674-680

29. Tena N, Lazzez A, Aparicio-Ruiz R, García-González DL (2007) Volatile Compounds Characterizing Tunisian Chemlali and Chétoui Virgin Olive Oils. J Agric Food Chem 55:7852-7858

30. Dias LA, Peres AM, Veloso ACA, Reis FS, Vilas-Boas M, Machado AASC (2009) An electronic tongue taste evaluation: Identification of goat milk adulteration with bovine milk. Sens Actuator B 136:209-217

31. Kobayashi Y, Habara M, Ikezazki H, Chen R, Naito Y, Toko K (2010) Advanced taste sensors based on artificial lipids with global selectivity to basic taste qualities and high correlation to sensory scores. Sensors 10:3411-3443
32. Bertsimas D, Tsitsiklis J (1992) Simulated annealing. Stat Sci 8:10-15

33. Cadima J, Cerdeira JO, Minhoto M (2004) Computational aspects of algorithms for variable selection in the context of principal components. Comput Stat Data Anal 47:225-236

34. Kirkpatrick S, Gelatt CD, Vecchi MP (1983) Optimization by simulated annealing. Science 220:671-680

35. Kuhn M, Johnson K (2013) Applied predictive modeling. Springer Science Business Media, New York

36. Cadima J, Cerdeira JO, Silva PD, Minhoto M (2012) The subselect R package. http://cran.rproject.org/web/packages/subselect/ vignettes/subselect.pdf. Accessed 15 Feb 2016

37. Venables WN, Ripley BD (2002) Modern Applied Statistics with $\mathrm{S}$ (Statistics and Computing), 4th edn. Springer, New York

38. Carrasco-Pancorbo A, Gómez-Caravaca AM, Cerretani L, Bendini A, Segura-Carretero A, Fernández-Gutiérrez A (2006) Rapid quantification of the phenolic fraction of Spanish virgin olive oils by capillary electrophoresis with UV detection. J Agric Food Chem 54:7984-7991

39. García A, Brenes M, García P, Romero C, Garrido A (2003) Phenolic content of commercial olive oils. Eur Food Res Technol 216:520-525

40. Romero C, Brenes M (2012) Analysis of total contents of hydroxytyrosol and tyrosol in olive oils. J Agric Food Chem 60:9017-9022

41. Dias LG, Sequeira C, Veloso ACA, Sousa MEBC, Peres AM (2014) Evaluation of healthy and sensory indexes of sweetened beverages using an electronic tongue. Anal Chim Acta 848:32-42

42. Panchuk V, Lvova L, Kirsanov D, Gonçalves CG, Di Natale C, Paolesse R, Legin A (2016) Extending electronic tongue calibration lifetime through mathematical drift correction: Case study of microcystin toxicity analysis in waters. Sens Actuator B 237:962-968

43. Correia DPA, Magalhães JMCS, Machado AASC (2005) Array of potentiometric sensors for simultaneous analysis of urea and potassium. Talanta 67:773-782

44. Apetrei C, Rodríguez-Méndez ML, de Saja JA (2005) Modified carbon paste electrodes for discrimination of vegetable oils. Sens Actuat B 111-112:403-409 